\title{
Acquired cognitive impairment in the presenium
}

\author{
R.C. Baldwin
}

\begin{abstract}
In a catchment area survey, 43 patients with cognitive impaiment acquired in the presenium associated with behovioural or social dystunction, or both, were identified. Twelve of 18 carers were also interviewed. The patients were mainly in their 50s, had a high prevalence of behavioural as opposed to cognitive disorder, were frequently taking psychotroplc drugs and had disorders which were largely progresstve. Those living at home wore as discbled as those living in residential seltings. Carers were stressed but uncomplaining. The findings suggest that poychiatists, perhaps especially old age peychiatitists, have an important role in developing services for this neglected group of people.
\end{abstract}

Presenile dementia is a rare disorder with many causes. The annual incidence of even the commonest cause, Alzheimer's disease (AD), is only 3.5-5.6 cases per 100,000 persons aged between 40 and 64 per annum (Newens et al, 1993a). A shortfall in service provision has been suggested (ADS, 1992), but very little is known about the needs of such individuals. Psychiatrists, in both general adult and specialist elderly services, are quite often approached to manage such patients and many express reservations.

This study describes a local survey of need and dependency among individuals with cognitive impairment in the presenium and of their carers. One of the aims was to examine the appropriateness or otherwise of involvement by psychiatric services.

\section{The study}

Entry criteria were:

(a) acquired cognitive impairment (ACI) in someone aged less than 65 years; $\mathrm{ACI}$ was defined as an impairment in cognition arising after the age of 16 confirmed by neuropsychological assessment and appropriate clinical and radiological investigation

(b) resident within Central Manchester Health Authority area

(c) a minimum of six months duration

(d) associated social or behavioural disability, or both, as judged by the treating clinician.

Local consultants, general practitioners, the departments of physiotherapy, speech therapy and occupational therapy, the Public Health Department and the Alzheimer Disease Society (ADS) were invited to submit names; a reminder was sent after two weeks.

The psychiatric and medical records of those so identified were examined (by RCB) to ascertain: the diagnosis; neuropsychological test findings; investigations; who had made the diagnosis; and the likely course of the disorder.

Eligible patients and their principal carer were interviewed using a semi-structured interview and the Clifton Assessment Procedure for the Elderly (CAPE) (Pattie \& Gilleard, 1979); the Mini-Mental State Examination (MMSE) (Folstein et al, 1975); the 28-item General Health Questionnaire (GHQ) (Goldberg, 1978) - the latter to carers. Enquiry was also made with local social services to establish whether any services were being provided. The census period covered three months.

\section{Findings}

The response rate was $76 \%$ and included all psychiatrists, neurologists, the ADS and all paramedical specialties. Of patients referred by GPs, only one was not already known to hospital services.

Sixty-five names were submitted. Twenty-two cases were excluded. In one the referrer advised against approaching the patient. Five were not living in Central Manchester; two had passed their 65th birthday; for one, case-notes were missing and one patient had died prior to the census period. Twelve were excluded because examination of the case-notes revealed insufficient or no evidence of cognitive impairment. The diagnoses of these patients were basal ganglia haemorrhage (1); basal ganglia calcification (1): alcohol and/or drug withdrawal states (4); epilepsy (1), 'generalised arteriopathy' (1); post head injury with recovery (2); metabolic encephalopathy (1); no clear neurological or psychiatric diagnosis (1).

Of the 43 patients who met the entry criteria. all but one had documented acquired neuropsychological deficits. The exception was one man 
with a clinical diagnosis of alcoholic dementia. He was living in a nursing home because of his impairments and was thus included. All but three patients had received neuroimaging.

It was possible to interview 32 of the subjects (74\%) with their carer or, if too impaired, the principal carer alone was interviewed.

\section{Demographic findings}

The median age was 59 (range 25-64 years). The age bands were $25-34=6 ; 35-44=5 ; 45-54=7$; $55-64=25$. There were 28 males and 15 females. Although only four (10\%) patients were engaged in any kind of occupation, paid or otherwise, all but two (5\%) had some kind of employment prior to their illness. Thirty (67\%) patients lived in their own homes, eight in nursing homes (19\%) and three in hospital long-stay wards (7\%); (other three [7\%]).

\section{Diagnoses and treatment}

The diagnoses were Alzheimer's disease $3(7 \%)$; multi-infarct dementia 14 (33\%); lobar atrophy (for example, frontal lobe dementia) 5 (11\%); alcohol-related cognitive impairment (including dementia) 8 (19\%); traumatic dementia $3(7 \%)$; post-stroke cognitive impairment 3 (7\%); Huntington's chorea $3(9 \%)$; other $4(7 \%)$. The expected outcome was stable disability 7 (16\%); progressive worsening 29 (68\%); fluctuating course 7 (16\%).

Medication received was none (20\%); neuroleptics (41\%); drugs for medical conditions (28\%); antidepressants (8\%); benzodiazepines (3\%).

\section{Dependency and impairment}

The median Mini-Mental State Examination score was 19 (range 0-29; missing cases=14). Of eight cases with MMSE scores greater or equal to 24 (the usually accepted cut-off for dementia), five had Clifton (CAPE) dependency ratings of grade $\mathrm{C}$ or worse, meaning at least moderately severe impairment.

The CAPE gives five levels of dependency: 'A' being independent through to $\mathrm{E}$, maximum dependency; typical of long-stay psychogeriatric wards. CAPE dependency levels are subdivided into: cognitlve dependency and behavioural dependency. Proportionately more patients had dependency grades of D or E on the behvaioural as opposed to the cognitive subsection $(35 \% \mathrm{v}$. 4\%).

Dependency levels of those living at home were compared with those in nursing homes. Although a third of cases could not be assessed on the CAPE cognitive portion (because it requires full cooperation of the patient), there were no significant differences in levels of dependency between these two groups on either the cognitive or behavioural CAPE subscales.

\section{Carers}

Fourteen patients (34\%) were living alone; 14 (32\%) with a relative; $11(25 \%)$ in an institution; and $4(9 \%)$ in other settings. Eighteen (41\%) had a 'whole-time' carer (defined as care equivalent to at least four days per week). Of these 18, 12 completed the 28-item General Health Questionnaire (GHQ) and seven scored above the accepted cut-off (4 out of 5) for psychiatric 'caseness'. None considered themselves emotionally ill (GHQ question 'do you consider yourself to have a nervous illness?') or had requested professional help.

\section{Services recetved}

Of those living in the community, no patients received services from statutory services (e.g. Meals on Wheels, home help services) or from mental health carers. Only five were in touch with community psychiatric nurses (CPNs). Only three patients attended a day centre or day hospital and only nine patients were in touch with a social worker.

\section{Comment}

More patients with acquired cognitive impairment, and associated social or behavioural disability, or both, in the presenium were identified than expected (catchment area of 120,000). This is probably due to deliberately broad entry criteria used ('acquired cognitive impairment' ACI - instead of operationally defined presenile dementia). This seems justified given the wide range of diagnoses uncovered. The finding of only three cases of Alzheimer's disease is surprising. Ten would have been expected applying the formula of Newens et al (1993b). Those from Afro-Caribbean and Asian ethnic groups were also under-represented in the survey, which itself was limited to patients known to primary or secondary health care over a limited census period of three months. Thus the true prevalence of ACI may have been higher.

The overall interview rate at three quarters is high enough to permit some conclusions which might serve to stimulate thought about appropriate models of care for this disparate group. First, the majority of patients were living in the community. They were as disabled as those in nursing homes, yet few received services from statutory or voluntary providers. Only a quarter had contact with a social worker. Unsurprisingly then, of those carers interviewed, a majority were stressed and poorly supported. 
Second, the community location of most of the patients and the comments made by carers, which were recorded at interview, did not support a need for a large in-patient base for this client group, either for assessment purposes or continuing care. Rather, the expressed needs were for coordination of hospital services (particularly neurologists who in this study most often made the diagnosis and psychiatrists who provided most of the basic out-patient care) with those in the community, especially planned, flexible social provision. Particular service shortfalls high-lighted were a lack of day care and welfare rights and benefits advice.

Third, the greatest burden, in terms of morbidity, as assessed by the CAPE, arose from behavloural disturbance rather than from cognitive impairment. This plus the high use of psychotropic medication (around 50\%) argues for psychiatric expertise in management. Specifically, given that most of the disorders were progressive in nature, the multidisciplinary, communityoriented model of old age psychiatry services may be the most relevant, or perhaps the least inappropriate service. Almost all old age psychiatric services already accept responsibility for a proportion of such patients. Whether other specialties, or even the patients and carers themselves, would agree is open to question (Shakespeare \& Anderson, 1992). However, in some areas, the lack of response by other professionals, including neurologists, neuropsychiatrists, neuropsychologists and neurorehabilitation experts, has resulted in local people voting with their feet; for example, in Liverpool a highly regarded community and day care service is coordinated by an old age psychiatrist. Surely it is time for the medical profession to begin a dialogue about how best to provide services to this neglected group.

\section{Acknowledgements}

The author wishes to thank Jane Turner for carrying out the interviews and to Janssen Pharmaceuticals for funding her.

\section{References}

AlzhEIMER'S Disease SOCIETY (1992) The Younger Person with Dementia Alzheimer's Disease Society. London: ADS. FolsteIN, M.F.. FolsteIn, S.E. \& MCHUGH, P.R. (1975) "Mini-Mental State": a practical method for grading the cognitive state of patients for the clinician. Journal of Psychiatric Research, 12, 185-198.

GolDBERG, D.P. (1978) Manual for the General Health Ques thonnaire. Windsor NFER-Nelson.

NEWENS, A.J., FORSTER, D.P., KAY, D.W.W. \& EDWARDSON, J. (1993a) Presenile dementia. British Medical Journal, s06, 1343.

-, FORSTER, D.P., KAY, D.W.K., KIRKUP, W., BATES, D. \& EDWARDSON, G. (1993b) Clinically diagnosed presentle dementia of the Alzheimer type in the Northern Health Region: ascertainment, prevalence, incidence and survival. Psychological Medicine, 23, 631-644.

PATTE, A.H. \& GILLEARD, C.J. (1979) Manual of the Clifton Assessment Procedures for the Elderly (CAPE). Sevenoaks: Hodder \& Stoughton.

SHAKESPEARE, J. \& ANDERSON, J. (1992) Huntington's disease - falling through the net. Health Trends, 28, 1923.

R.C. Baldwin, Consultant Psychiatrist for the Elderly, York House, Manchester Royal Infirmary. Manchester M13 9BX 